\title{
B7-H1 protein vaccine induces protective and therapeutic antitumor responses in SP2/0 myeloma-bearing mice
}

\author{
CUN ZHANG ${ }^{*}$, WEIHUA WANG* ${ }^{*}$ XIN QIN, YUJIN XU, TONGLIE HUANG, \\ QIANG HAO, WEINA LI, SHOUZHEN WU and YINGQI ZHANG

\begin{abstract}
State Key Laboratory of Cancer Biology, Biotechnology Center, School of Pharmacy, The Fourth Military Medical University, Xi'an, Shaanxi 710032, P.R. China
\end{abstract}

Received January 15, 2013; Accepted March 19, 2013

DOI: $10.3892 /$ or.2013.2686

\begin{abstract}
B7-H1 is a co-inhibitory molecule belonging to the B7 family. The B7-H1 protein is only expressed on macrophage lineage of cells in normal tissues, but is overexpressed in most types of tumor. The aberrant expression of cell surface B7-H1 on cancer cells is generally associated with high-risk prognostic factors. The tumor-associated B7-H1 increases apoptosis of antigen-specific $\mathrm{T}$ cells through interaction with its receptor $\mathrm{PD}-1$ on $\mathrm{CD}^{+} \mathrm{T}$ cells and contributes to tumor immune evasion. These features suggest that B7-H1 may be a therapeutic target for the B7-H1-expressing tumors. We developed a therapeutic vaccine by coupling a tetanus toxoid T-helper cell epitope with the N-terminal of B7-H1 IgV-like domain. This vaccine was able to induce high titers of antibodies against $\mathrm{B} 7-\mathrm{H} 1$ in mice which were able to bind to native cell surface B7-H1. We chose the B7-H1-expressing SP2/0 myeloma and its syngeneic host (the BALB/c mouse) as the model to study the antitumor activity of the rhB7-H1M vaccine. Vaccination with this modified $\mathrm{B} 7-\mathrm{H} 1$ protein resulted in almost complete protection from SP2/0 tumor challenge and efficiently eliminated pre-established tumors in mice. In addition, B7-H1 vaccination was able to decrease the percentage of $\mathrm{CD}^{+}{ }^{+} \mathrm{Foxp}^{+}$regulatory $\mathrm{T}$ cells in tumor-bearing mice and which might improve antitumor immunity. These data demonstrate the potential of B7-H1-based vaccine as a therapeutic agent for the treatment of cancer overexpressing B7-H1.
\end{abstract}

\section{Introduction}

B7-H1, also known as programmed death ligand-1 (PD-L1) or CD274, is a co-inhibitory member of the B7 family (1).

Correspondence to: Professor Yingqi Zhang, State Key Laboratory of Cancer Biology, Biotechnology Center, School of Pharmacy, The Fourth Military Medical University, 17 Changle West Road, Xi'an, Shaanxi 710032, P.R. China

E-mail: zhangyqh@fmmu.edu.cn

\section{${ }^{*}$ Contributed equally}

Key words: B7-H1, vaccine, immune evasion, tetanus toxoid T-helper epitope, $\mathrm{CD}^{+}{ }^{+} \mathrm{Foxp}^{+}$regulatory $\mathrm{T}$ cells
The B7-H1 protein is a cell surface glycoprotein which is only expressed on macrophage lineage of cells in normal tissues (1-2). The immunoglobulin (Ig)V-like domain of B7-H1 plays an essential role in the interaction with its receptor (3). The receptor for B7-H1 is programmed death-1 (PD-1, CD279), which is a co-inhibitory receptor (4). In addition to PD-1, other receptors for B7-H1 may exist (4). The ligation of B7-H1 on antigen-presenting cells (APC) with PD-1 on T cells induces anergy in naive $\mathrm{T}$ cells and apoptosis in effector T cells (1). Thus, it is now well accepted that B7-H1 is a negative regulator of $\mathrm{T}$ cell immunity.

$\mathrm{B} 7-\mathrm{H} 1$ protein is overexpressed in various malignancies including lung (5), brain (6), melanoma (3), breast (7-8), and renal (9) cancer. Furthermore, a correlation between the expression of B7-H1 on cancer cells and patient clinic pathological status has been shown in some of these malignancies (7-9). Ligation of B7-H1 on cancer cells with PD-1 on tumor-specific $\mathrm{T}$ cells has been demonstrated to suppress $\mathrm{T}$-cell activation and proliferation, and to induce T-cell apoptosis (10). Tumor cells exploit this regulatory interaction as a mechanism of immune evasion. Furthermore, cell surface B7-H1 could serve as a receptor to protect cancer cells from apoptosis induced by Fas binding or the protein kinase inhibitor staurosporine (11).

Aside from tumor cells, some immune cells in the tumor microenvironment also expressed B7-H1, including macrophages and dendritic cells (DCs) (12). Cell surface B7-H1 on DCs is involved in the induction and maintenance of T-cell anergy (12). Signaling through B7-H1 on DCs is required for the induction of adaptive $\mathrm{CD} 4^{+} \mathrm{Foxp}^{+}$regulatory $\mathrm{T}$ cells $\left(\mathrm{CD}^{+}{ }^{+}\right.$Foxp $3^{+}$Treg) and the modulation $\mathrm{CD}^{+}{ }^{+}$Foxp $3^{+}$Treg cell-mediated immune inhibition (13). It is significant that the induced Tregs were able to suppress antitumor immunity $(14,15)$. Thus, B7-H1 may play an important role in the immune evasion from the host immune system in cancer patients.

B7-H1 may be regarded as a tumor associated antigen as well as an immune-suppressor playing an important role in the immune escape of tumor cells. Blockade of the B7-H1 signal pathway may represent novel strategies to inhibit tumor growth and enhance T-cell tumor immunity in cancer. Previous study showed that administration of mouse B7-H1 monoclonal antibody inhibited the growth of B7-H1/P815 in vivo (3). Moreover, blockade of the B7-H1/PD-1 pathway with soluble 
PD-1-IgV protein was able to enhance CTL activity and antitumor effects on tumor-bearing mice (16).

Active vaccination against B7-H1 is an approach that may present advantages over passive $\mathrm{mAb}$ therapy by inducing a polyclonal $\mathrm{Ab}$ response, potentially leading to better activation of Ab-dependent effector functions. However, the efficacy of active vaccination may be limited by the fact that B7-H1 is a self $\mathrm{Ag}$ with poor immunogenicity. In this study, insertion of foreign T-helper cell epitope (17) was designed to bypass B7-H1-specific CD4 ${ }^{+}$Th tolerance. The approach of such a Th chimeric vaccine was effective toward self Ags in mice, such as the proinflammatory cytokine TNF- $\alpha$ (18), B-lymphocyte stimulator (BLyS) (19), and HER2 (20).

In this study, we coupled the P2 tetanus toxoid T-helper epitope (QYIKANSKFIGITE) $(21,22)$ to the $\mathrm{N}$ terminal end of B7-H1 IgV-like domain (known as recombinant human B7-H1 mutant, rhB7-H1M). The result demonstrated that the rhB7-H1M protein vaccination was highly effective in both preventive and therapeutic animal models.

\section{Materials and methods}

Construction, expression and purification of $r h B 7-H 1 M$ proteins. The rhB7-H1M gene fragment was commercially synthesized according to the sequence of human B7-H1 in the NCBI databank (gi:20070268) (Bioasia Company, China). The DNA fragment was then cloned into plasmid pQE-30 (Qiagen, Germany). rhB7-H1M protein was expressed in E. coli by isopropyl-1-thio- $\beta$-galactopyranoside (IPTG) induction. The isolated inclusion bodies were resuspended in solubilizing buffer B with $6 \mathrm{~mol} / \mathrm{l}$ urea. The supernatant was applied to the $\mathrm{Ni}^{2+}$ chelate affinity column. Bound proteins were eluted with $250 \mathrm{mmol} / \mathrm{l}$ imidazole in buffer B and refolded by dialyzing against buffers with decreasing denaturant in $20 \mathrm{mmol} / \mathrm{l}$ Tris- $\mathrm{HCl}$ (pH 9.0): first $4 \mathrm{~mol} / \mathrm{l}$ urea, then $2 \mathrm{~mol} / \mathrm{l}$ urea and $1 \mathrm{mmol} / \mathrm{l}$ EDTA, and finally PBS ( $\mathrm{pH}$ 7.4). The protein was characterized by SDS-PAGE, western blotting, size exclusion chromatography high performance liquid chromatography (SEC-HPLC).

SEC-HPLC analysis. SEC-HPLC analysis was performed on a Beckman's HPLC system (Beckman Coulter, USA). The protein sample in PBS was injected onto a $7.5 \times 300 \mathrm{~mm}$ G2000SW column (Tosoh, Japan). Peaks were detected by monitoring at a wavelength of $280 \mathrm{~nm}$. The purity of the rhB7-H1M protein was calculated as a percentage of the total peak area detected.

Mice, cell lines, and tumor challenges. Healthy female BALB/c mice (6-8 weeks old, purchased from the National Rodent Laboratory Animal Resource, Shanghai, China) were cared for under institutional animal care protocols in the Experimental Animal Center of the Fourth Military Medical University.

SP2/0 (mouse myeloma cell line) and HT-29 (human colorectal carcinoma cell line) were maintained in RPMI-1640 medium (Sigma-Aldrich, USA). HT-29/B7-H1-expressing cell line was obtained by stimulating with $20 \mathrm{ng} / \mathrm{ml}$ human interferon- $\gamma($ IFN- $\gamma)($ R\&D Systems, USA) for $48 \mathrm{~h}$.

For injection, tumor cells were harvested, washed and suspended at the desired concentration in serum-free medium.
Mice were injected s.c. in the lower back with $100 \mu \mathrm{l}$ cell suspension. Tumor growth was measured with calipers and is presented as the average of the products of two bisecting diameters.

Immunizations. Proteins for immunization were emulsified 1:1 (v/v) in IFA (Sigma-Aldrich). Mice were injected s.c. in the neck region with $40 \mu \mathrm{g}$ of protein in a total volume of $100 \mu \mathrm{l}$. Unless otherwise stated, vaccinations were performed every other week for a total of three times.

Flow cytometry. To determine the antigen-binding capacity of antiserum, HT-29/B7-H1(+) and SP2/0 cells were incubated with antiserum samples collected from mice vaccinated by rhB7-H1M, anti-B7-H1 mAb (positive control), or normal serum (vaccinated by adjuvant, negative control) and analyzed by the flow cytometer (Becton-Dickinson, USA). For blocking the binding capacity of mouse serum, tumor cells were incubated with the antiserum samples which were pretreated with $\mathrm{B} 7-\mathrm{H} 1 / \mathrm{Fc}$ for $30 \mathrm{~min}$.

To identify the changes of $\mathrm{CD}^{+}{ }^{+} \mathrm{Foxp}^{+} \mathrm{T}$ cells, lymph cells from mouse peripheral blood were incubated with anti-CD4 phycoerythrin (PE), anti-Foxp3-fluorescein isothiocyanate (FITC), or appropriate isotype controls (eBioscience, USA), and then analyzed by flow cytometry.

Complement-dependent cytotoxicity (CDC) assay. The SP2/0 cells were washed and $50 \mu \mathrm{l}$ of $1 \times 10^{6}$ cells $/ \mathrm{ml}$ were plated per well in 96-well plates (Costar, USA). Cells were incubated with antibody or antiserum diluted 1:10 with normal guinea pig serum (used as complement). MTT was added. The plate was incubated for $4 \mathrm{~h}$ and DMSO was added. The OD was measured at $570 \mathrm{~nm}$. Anti-B7-H1mAb $(0.1 \mu \mathrm{g} /$ well $)$ was used as a positive control. The experiments were repeated at least three times.

ELISPOT assay. Splenocytes from vaccinated mice were isolated and restimulated with B7-H1-expressing SP2/0 cells (splenocytes:tumor cells, 30:1) and cultured at $37^{\circ} \mathrm{C}$ with $5 \%$ $\mathrm{CO}_{2}$ for $24 \mathrm{~h}$. SP2/0 cells were killed by $60 \mathrm{~Gy}$ irradiation and immediately used as antigen presenting cells specifically for B7-H1. The restimulated splenocytes were then added to antimouse IFN- $\gamma$ mAb precoated 96-well plates $\left(2.5 \times 10^{5} /\right.$ well) and further incubated at $37^{\circ} \mathrm{C}$ with $7 \% \mathrm{CO}_{2}$ for $4 \mathrm{~h}$. The experiment was performed according to the manufacturer's instructions (IFN- $\gamma$ ELISPOT kit from Invitrogen, USA). The spots were counted using a dissecting microscope. The spot numbers were the mean of triplicates in each vaccinated group.

Passive transfer. Sera from BALB/c mice vaccinated with rhB7-H1M were pooled, and the B7-H1-specific Ab concentration was determined by ELISA using a standard curve. A total of 100-200 $\mu \mathrm{l}$ of the pooled sera was injected into the peritoneum of naive BALB/c mice 1 day before s.c. challenge with $5 \times 10^{6} \mathrm{SP} 2 / 0$ tumor cells. Control animals were injected with $500 \mu \mathrm{g}$ nonspecific mouse IgG.

Statistical analysis. All data were analyzed by Student's t-test. Results are expressed as the means \pm standard deviations (SD). A P-value of $<0.05$ was considered to indicate a statistically significant difference. 


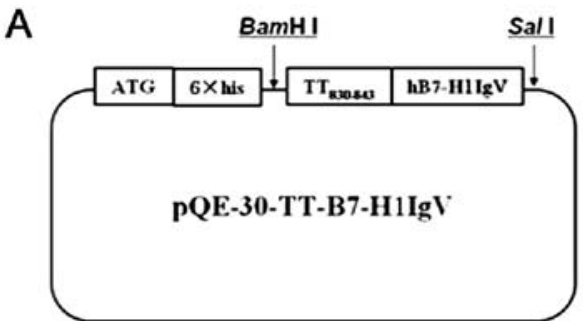

C

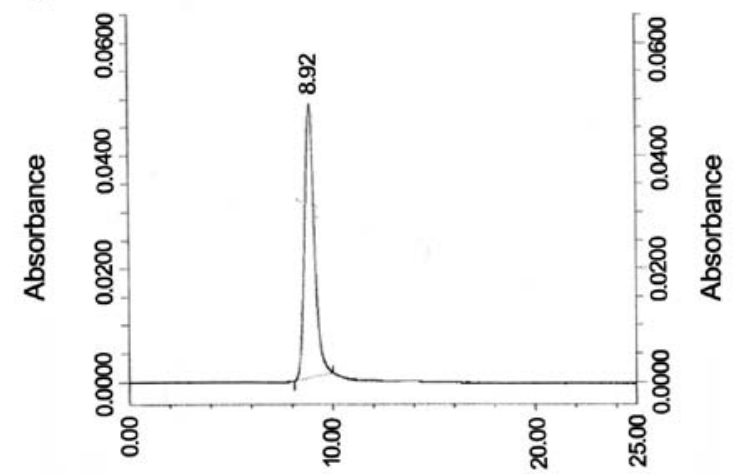

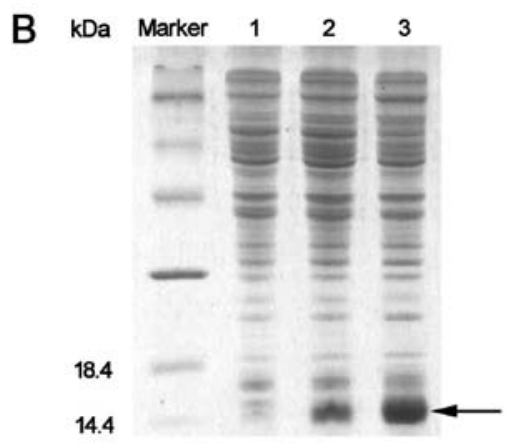

D

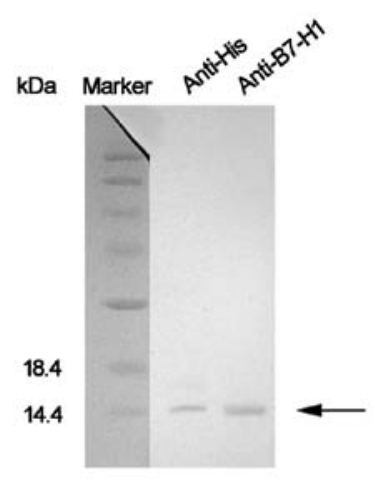

Figure 1. The recombinant molecule rhB7-H1M and characterization of B7-H1 expression in cancer cell lines. (A) Schematic representation of the rhB7-H1M coding region in pQE30. (B) Bacterial expression of rhB7-H1M was analyzed by SDS-PAGE. Lane 1, uninduced M15 cell lysate of pQE30-rhB7-H1M; lane 2, rhB7-H1M M15 cell lysates; lane 3, inclusion body of rhB7-H1M. (C) HPLC analysis of renatured fusion protein. (D) Western blot analysis of the renatured protein using anti-His and anti-hB7-H1 antibodies.

\section{Results}

rhB7-H1M protein is expressed and purified from E. coli. The DNA fragments encoding rhB7-H1M were cloned into the vector, pQE30 (Fig. 1A). The recombinant plasmid was transformed into $E$. coli to express the fusion protein with an $\mathrm{N}$-terminal six-histidine tag. The IPTG-induced target proteins were detected by SDS-PAGE. The observed molecular weight of rhB7-H1M was $\sim 15 \mathrm{kDa}$, consistent with the expected size. These fusion proteins, mainly existing in inclusion bodies (Fig. 1B, lane 3), were solubilized and purified by $\mathrm{Ni}^{2+}$ chelate affinity chromatography under denaturing conditions. The protein was then refolded by dialysis. The purity of the target protein was at least $95 \%$ as identified by HPLC (Fig. 1C). The recombinant protein was further analyzed by western blotting with anti-His and anti-hB7-H1 antibodies (Fig. 1D).

rhB7-H1M protein vaccine mediates tumor protection in mice. The rhB7-H1M protein vaccine was evaluated for the ability to elicit a protective immune response against challenge with a syngeneic B7-H1-expressing SP2/0 tumor cell line. Two groups of $10 \mathrm{BALB} / \mathrm{c}$ mice were vaccinated with either rhB7H1M protein or with adjuvant, negative control, respectively. Two weeks later, the mice were challenged with $5 \times 10^{6} \mathrm{SP} 2 / 0$ cells and tumor growth was monitored. The mice vaccinated with adjuvant, negative control developed large solid tumors within 12-20 days of subcutaneous administration. Vaccination with the rhB7-H1M protein significantly inhibited the growth of the tumor cells (Fig. 2A) and only 40\% (4/10) of the mice developed small, slow growing tumors. Thirty-six days after the challenge, the mice were sacrificed and if a solid tumor was present, its mass was determined. In the group vaccinated with the rhB7-H1M protein, the mean mass of tumors from only four mice was significantly less than the mean mass of the tumors in the adjuvant, negative control group (1.84 vs. $6.90 \mathrm{~g} ; \mathrm{P}<0.05)$. In addition, the life span of another two groups of BALB/c mice with the same treatment as above was observed. The increase in survival rate in mice vaccinated with the rhB7-H1M protein was also statistically significant $(\mathrm{P}<0.05)$ (Fig. 2B).

rhB7-HIM protein vaccine treats established tumors. To test the therapeutic efficacy of the vaccine in a situation that more closely resembles the clinical setting, we allowed tumors to establish before vaccinating with the rhB7-H1M protein. At Day $0, \mathrm{BALB} / \mathrm{c}$ mice were inoculated with $5 \times 10^{6} \mathrm{SP} 2 / 0$ cells. At Day 7, the mice were immunized with rhB7-H1M protein or adjuvant, negative control. Treatment with the rhB7-H1M protein had a therapeutic effect on tumor growth (Fig. 3A). The average tumor growth rate was decreased in some instances. Similarly, tumor incidence was reduced in the rhB7-H1M group, as 30\% (3/10) of rhB7-H1M vaccinated mice remained tumor-free, vs. $0 \%$ ( 0 of 10 ) of adjuvant, negative control mice. Thirty-six days after the challenge, the mice were sacrificed. The tumor weight of the rhB7-H1M vaccination group was significantly lower $(3.30 \pm 0.76 \mathrm{~g})$ than that of the adjuvant, negative control group $(6.98 \pm 1.01 \mathrm{~g})(\mathrm{P}<0.05)$. Furthermore, we observed statistically significant differences of survival time in another two groups of BALB/c mice with the same 

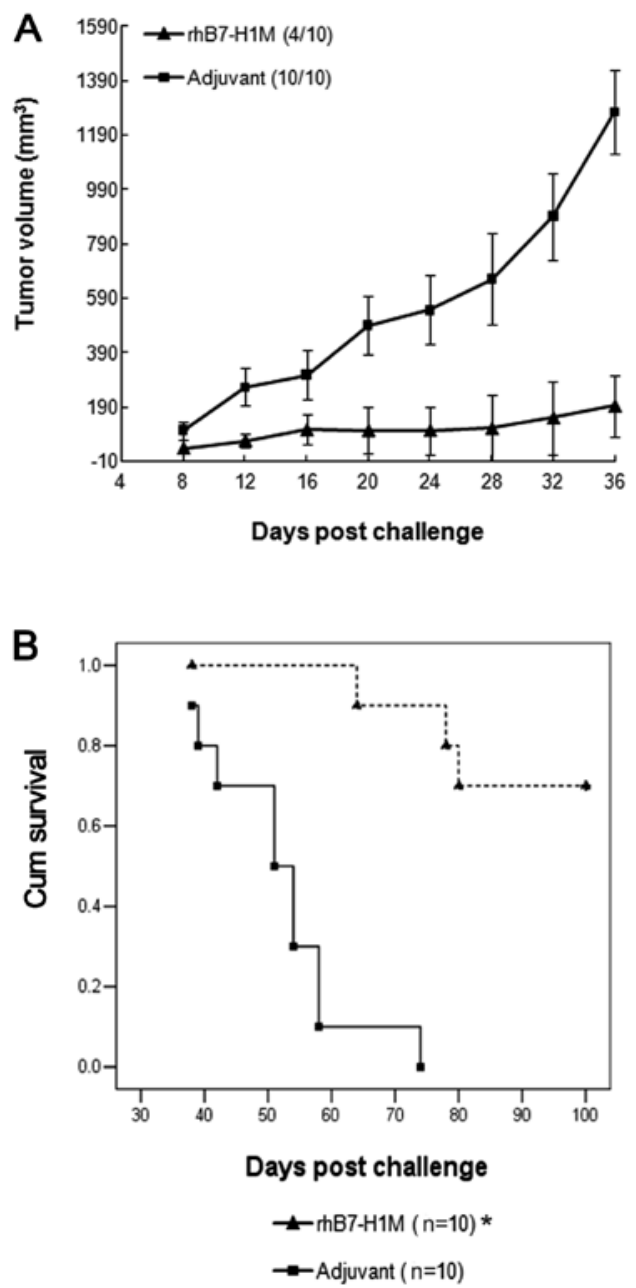

Figure 2. SP2/0 tumor protection mediated by the rhB7-H1M protein vaccine in BALB/c mice. (A) Growth of SP2/0 cells was almost completely inhibited by vaccination with the rhB7-H1M protein. The number of animals that developed tumors/total number of animals is indicated in parenthesis. (B) Life span of BALB/c mice after challenge of SP2/0 cells. The survival data were subjected to Kaplan-Meier analysis. "Statistically significant difference compared with the control adjuvant mice $(\mathrm{P}<0.05)$. The number in parentheses is the number of mice in the experiment.

treatment as above (Fig. 3B) and the rhB7-H1M vaccination prolonged the life span of tumor bearing mice $(\mathrm{P}<0.05)$.

Cellular and humoral immunity. To investigate the immunological mechanism underlying the therapeutic effect of the rhB7-H1M vaccine, we measured the anti-B7-H1 antibody in mouse serum. The vaccine elicited strong anti-hB7-H1 and anti-mB7-H1 antibody responses (Fig. 4A). However, there was almost no cross-binding activity of antiserum with B7-H4, which belongs to the same family as B7-H1. The results suggested that antibodies induced by the rhB7-H1M vaccine can interact with human as well as mouse B7-H1 antigens. The ability of the serum antibodies to recognize the native cell surface B7-H1 protein was tested by flow cytometry. The serum antibodies were able to bind to B7-H1-expressing HT-29 and SP2/0 cells (HT-29/B7-H1, 81.5\%; SP2/0, 77.5\%) (Fig. 4B). To determine binding specificity, rhB7-H1/Fc protein and $\mathrm{rmB} 7-\mathrm{H} 1 / \mathrm{Fc}$ protein were used to compete with the native membrane B7-H1 respectively. The competitive inhibition rates were 93.1 and $90.8 \%$, respectively (Fig. 4B).
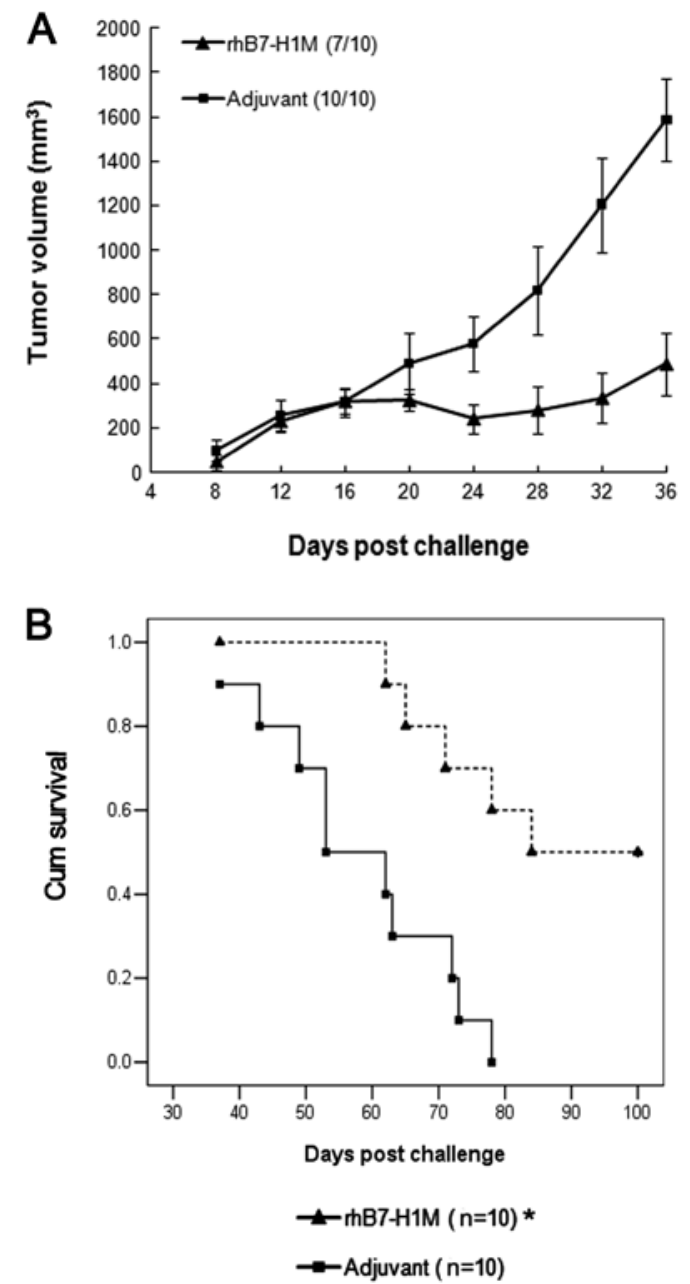

Figure 3. Therapeutic effects of rhB7-H1M protein vaccine on established $\mathrm{SP} 2 / 0$ tumor in BALB/c mice. (A) Growth of SP2/0 cells was partially inhibited by the rhB7-H1M protein vaccination. The number of animals that developed tumors/total number of animals is indicated in parenthesis. (B) Life span of BALB/c mice after challenge of SP2/0 cells. The survival data were subjected to Kaplan-Meier analysis. "Statistically significant difference compared with the control adjuvant mice $(\mathrm{P}<0.05)$. The number in parentheses is the number of mice in the experiment.

These data indicated that the rhB7-H1M vaccine resulted in the production of antibodies to a mixture of native structures.

In addition, antisera from BALB/c mice immunized with the rhB7-H1M protein were capable of depleting B7-H1expressing SP2/0 cells in vitro through CDC (Fig. 4C). The killing rate of SP2/0 cells reached $53.4 \pm 9.4 \%$ (1:1,000-fold diluted anti-B7-H1 mAb) and 48.7 $\pm 8.2 \%$ (1:1,000-fold diluted antiserum), respectively. The killing rate decreased as dilution of anti-B7-H1 mAb and antiserum.

To measure B7-H1-specific cellular immune response, the number of IFN- $\gamma$-producing B7-H1-specific T-cell precursors was determined by ELISPOT assay. There was a weak generation of IFN- $\gamma+$ spots stimulated in splenocytes from mice immunized with the rhB7-H1M protein vaccine (Fig. 4D). Therefore, it was suggested that cellular immunity may not play a major role in mediating therapeutic effects of the rhB7H1M protein vaccine.

Passive transfer of B7-HI-specific Abs protects naive mice from tumor challenge. The high $\mathrm{Ab}$ response and weak cellular 


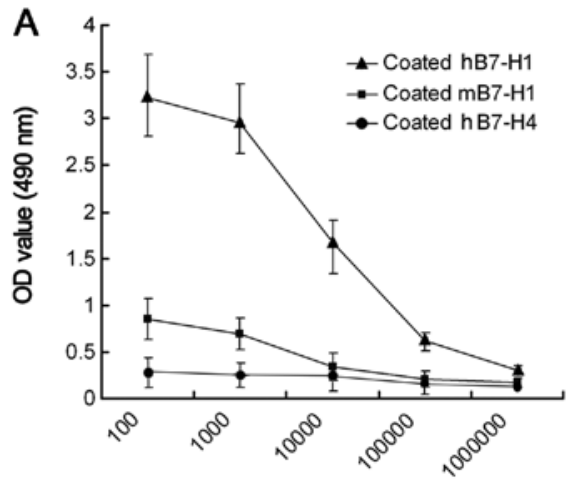

Dilution fold

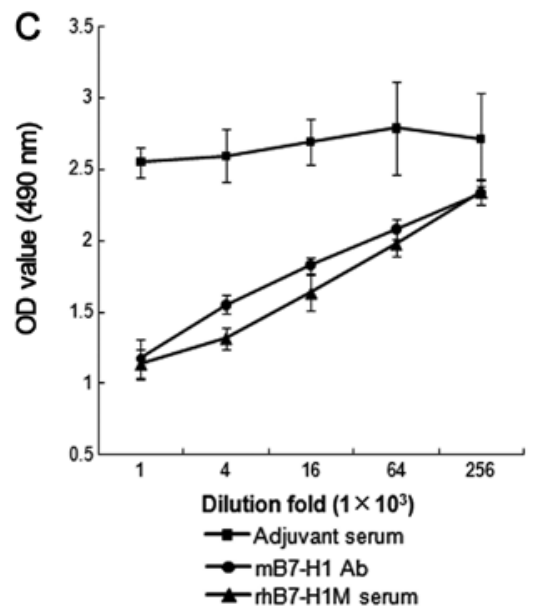

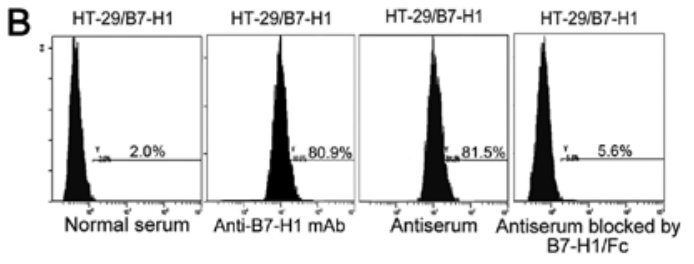

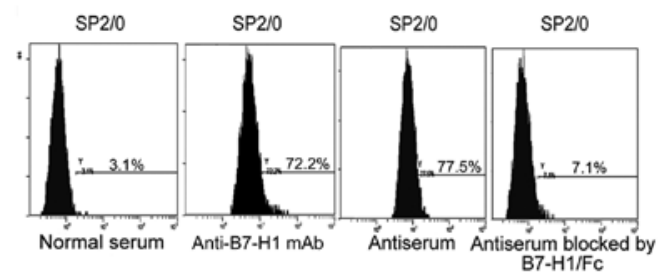

D

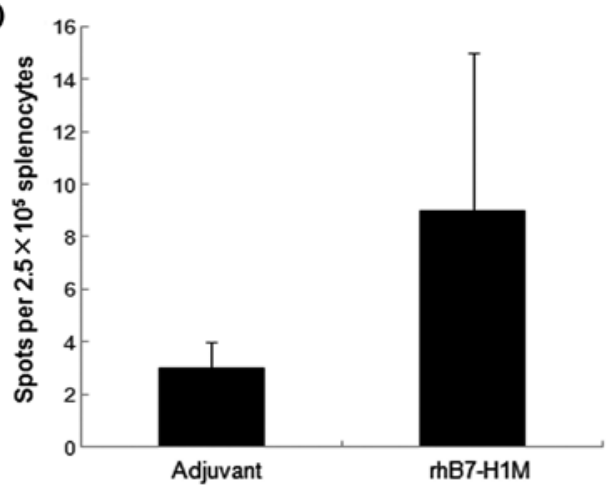

Figure 4. Immune responses induced by rhB7-H1M vaccination. (A) Ab responses in BALB/c mice vaccinated three times with $40 \mu \mathrm{g}$ rhB7-H1M protein in IFA. The figures show the titration curves of anti-B7-H1 sera against human B7-H1 protein or mouse B7-H1 protein. Human B7-H4 protein was used as negative control. (B) Flow cytometric analysis of the binding ability of anti-B7-H1 sera to native B7-H1 on cell surface. Anti-B7-H1 mAb was used as positive control and normal serum (vaccinated by adjuvant) as negative control. (C) Complement-mediated killing of SP2/0 tumor cells with serum from mice vaccinated with the rhB7-H1M protein. Anti-B7-H1 mAb was used as a positive control and the serum from adjuvant vaccination was used as a negative control. (D) Induction of B7-H1-specific IFN- $\gamma$-producing cellular immune responses by the rhB7-H1M vaccine. The number of IFN- $\gamma$-producing B7-H1-specific T-cell precursors was determined using ELISPOT assay. The data were analyzed using t-test. rhB7-H1M vaccine vs. adjuvant control P $>0.05$.

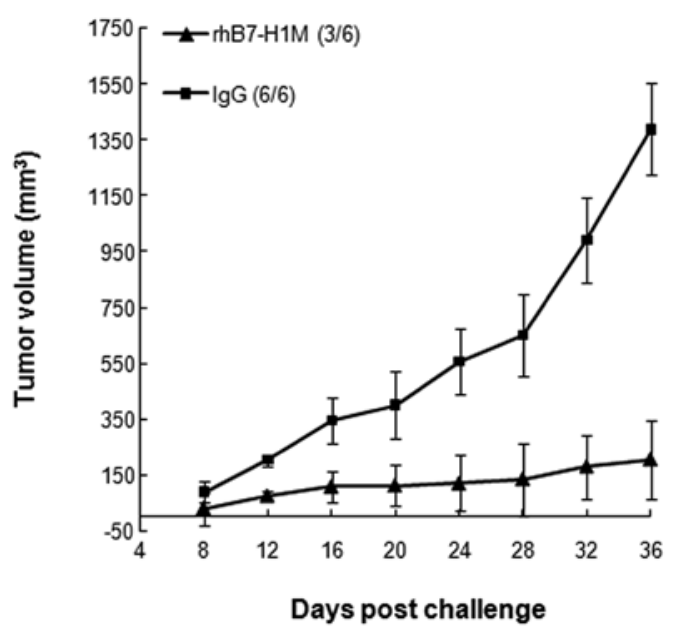

Figure 5. B7-H1-specific Abs conferred tumor protection. SP2/0 tumor growth in $\mathrm{BALB} / \mathrm{c}$ mice $(\mathrm{n}=6)$ treated with sera from rhB7-H1M protein-vaccinated mice (containing $1.6 \times 10^{6}$ units of B7-H1-specific Abs) or control IgG.

immune response suggested that Abs may be responsible for the inhibition of tumor growth. To confirm this, antisera from $\mathrm{BALB} / \mathrm{c}$ mice immunized with $\mathrm{rhB} 7-\mathrm{H} 1 \mathrm{M}$ protein

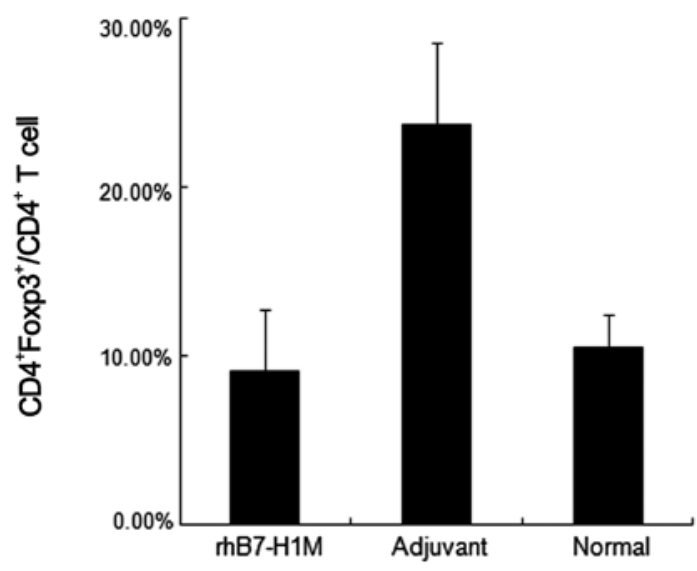

Figure 6. Flow cytometric analysis of the ratio of $\mathrm{CD} 4^{+} \mathrm{Foxp}^{+} \mathrm{T}$ cells to $\mathrm{CD} 4^{+} \mathrm{T}$ cells in peripheral blood of each group in a therapeutic mice model. The data were analyzed using t-test. rhB7-H1M vaccine vs. adjuvant control $\mathrm{P}<0.05$. Adjuvant control vs. normal control $\mathrm{P}<0.05$. rhB7-H1M vaccine vs. normal control $\mathrm{P}>0.05$.

were injected into the peritoneum of $\mathrm{BALB} / \mathrm{c}$ mice that were subsequently challenged with SP2/0 tumor cells. Tumor grew at normal rates in mice injected with control $\mathrm{IgG}$, while tumor 
growth rate was significantly reduced in mice that received B7-H1-specific antisera (Fig. 5). Collectively, these results suggest that the rhB7-H1M vaccine may mediate tumor rejection mainly through Abs induced by itself in this animal model.

rhB7-H1M vaccine reduces the level of $\mathrm{CD}^{+}{ }^{+}$Foxp $3^{+}$Treg cells in tumor-bearing mice. As discussed above, B7-H1 in the tumor microenvironment is involved in the induction of $\mathrm{CD}^{+}{ }^{+} \mathrm{Foxp}^{+}{ }^{+}$Treg through the B7-H1/PD-1 signal pathway. To identify whether the rhB7-H1M protein vaccine could block the induction of $\mathrm{CD}^{+}{ }^{+}$Foxp $3^{+}$Treg, we detected the ratio of CD $4^{+}$Foxp $3^{+}$Treg cells to $\mathrm{CD} 4^{+} \mathrm{T}$ cells in peripheral blood in the therapeutic mice models. Similarly, the mice in the vaccination group showed a significant decrease in the percentage of $\mathrm{CD}^{+}{ }^{+}$Foxp $3^{+}$Treg cells compared to the adjuvant, negative control group and the level of $\mathrm{CD}^{+}{ }^{+} \mathrm{Foxp} 3^{+}$Treg cells in the vaccination group were almost equal to the levels in normal mice (Fig. 6).

\section{Discussion}

The molecule of the B7 family belongs to the immunoglobulin (Ig) superfamily. The IgV-like domain of this family is responsible for the interaction of ligands and receptors $(2,23)$. The sequence identity of the amino acids between human and mouse B7-H1 IgV domain is up to $91 \%$. In this study, the antiserum by rhB7-H1M vaccine can bind to native mouse and human B7-H1. It is indicated that the rhB7-H1M vaccine could break species restriction and induce cross-reactive Abs against B7-H1 IgV domain of mice and humans. Thus, functional studies by xenogeneic hB7-H1 vaccination are feasible in mice.

In this study, rhB7-H1M protein vaccines elicited high B7-H1-specific Ab titers and efficiently prevented transplantable tumor growth, and eradicated pre-established tumors in mice models. Together with passive transfer of Abs, it is suggested that Ab-mediated immune responses are critical in antitumor immunity induced by the rhB7-H1M protein vaccine. The effective therapeutic role for B7-H1-specific Abs was previously suggested by Iwai et al (10). In the present study, we observed that polyclonal anti-B7-H1 Abs could directly deplete B7-H1-expressing tumor cells through the CDC effect in vitro. The mechanisms of antibody involvement in the destruction of tumor cells in vivo require further investigation.

By contrast, lower cellular immune response appeared negligible and did not correlate with tumor protection. However, additional evidence is required to better understand the level of T-cell-mediated immunity induced by the rhB7-H1M vaccine. For example, whether depletion of $\mathrm{CD}^{+} \mathrm{T}$ cells or $\mathrm{CD}^{+} \mathrm{T}$ cells decreased the efficacy of antitumor immunity was not tested in the current study, but warrants further investigation.

In addition, inflammatory mediators such as IFN- $\gamma$ are implicated in the upregulation of B7-H1 expression in several tumor cell lines (24). T cells or natural killer cells in tumor tissue or tumor cells themselves secrete several cytokines, including IFN- $\gamma$. Therefore, one possible scenario is that TILs and tumor cells secrete IFN- $\gamma$ in the beginning, followed by upregulation of B7-H1 in tumor cells; thereafter, the upregulated $\mathrm{B} 7-\mathrm{H} 1$ induces $\mathrm{CD}{ }^{+} \mathrm{T}$-cell apoptosis through interaction with PD-1 (25). Finally, these factors make up a positive feedback circle in the tumor microenvironment to help tumor immune escape. In this study, Abs induced by rhB7-H1M vaccination may block B7-H1/PD-1 interaction in vivo. The blockade may destroy this circle and rescue T-cell function which ultimately translates into antitumor immunity (26).

As discussed above, B7-H1 is involved in inducing CD $4^{+}$Foxp $3{ }^{+}$Treg through the B7-H1/PD-1 pathway (11). CD $4^{+}$Foxp $3^{+}$Treg could induce anergy of tumor specific CTL. Thus, these negative immunoregulatory checkpoints form another network of tumor immune escape. Our preliminary results showed that rhB7-H1M vaccination could decrease the percentage of $\mathrm{CD}^{+}{ }^{+}$Foxp $3{ }^{+}$Treg cells in tumor-bearing mice ,although the mechanisms remain unknown. Thus, the rhB7$\mathrm{H} 1 \mathrm{M}$ vaccine may partly reverse the condition of immune suppression in a tumor microenvironment and expose tumor cells to the host antitumor immunity.

In summary, the present study demonstrated the utility of the rhB7-H1M protein vaccine is potentially an effective means of cancer therapy. Current studies are underway to evaluate the rhB7-H1M vaccine in primate models in an attempt to develop vaccines useful for treating human B7-H1expressing carcinomas.

\section{Acknowledgements}

The authors thank the members of the Biotechnology Center of the Fourth Military Medical University for their excellent technical support and we especially thank Dr Qiuyang Daniel Zhang for the suggestion on the experiment design. This study was funded by the National Natural Foundation of China, NSFC30900537.

\section{References}

1. Dong H, Zhu G, Tamada K and Chen L: B7-H1, a third member of the B7 family, co-stimulates T-cell proliferation and interleukin-10 secretion. Nat Med 5: 1365-1369, 1999.

2. Dong H, Strome SE, Salomao DR, et al: Tumor-associated B7-H1 promotes T-cell apoptosis: a potential mechanism of immune evasion. Nat Med 8: 793-800, 2002.

3. Hansen JD, Du Pasquier L, Lefranc MP, Lopez V, Benmansour A and Boudinot P: The B7 family of immunoregulatory receptors: a comparative and evolutionary perspective. Mol Immunol 46: 457-472, 2009.

4. Freeman GJ, Long AJ, Iwai Y, et al: Engagement of the PD-1 immunoinhibitory receptor by a novel B7 family member leads to negative regulation of lymphocyte activation. J Exp Med 192: 1027-1034, 2000.

5. Konishi J, Yamazaki K, Azuma M, Kinoshita I, Dosaka-Akita H and Nishimura M: B7-H1 expression on non-small cell lung cancer cells and its relationship with tumor-infiltrating lymphocytes and their PD-1 expression. Clin Cancer Res 10: 5094-5100, 2004.

6. Wintterle S, Schreiner B, Mitsdoerffer M, et al: Expression of the B7-related molecule B7-H1 by glioma cells: a potential mechanism of immune paralysis. Cancer Res 63: 7462-7467, 2003.

7. Ghebeh H, Mohammed S, Al-Omair A, et al: The B7-H1 (PD-L1) T lymphocyte-inhibitory molecule is expressed in breast cancer patients with infiltrating ductal carcinoma: correlation with important high-risk prognostic factors. Neoplasia 8: 190-198, 2006.

8. Hasan A, Ghebeh H, Lehe C, Ahmad R and Dermime S: Therapeutic targeting of B7-H1 in breast cancer. Expert Opin Ther Targets 15: 1211-1225, 2011.

9. Thompson RH, Kuntz SM, Leibovich BC, et al: Tumor B7-H1 is associated with poor prognosis in renal cell carcinoma patients with long-term follow-up. Cancer Res 66: 3381-3385, 2006.

10. Iwai Y, Ishida M, Tanaka Y, Okazaki T, Honjo T and Minato N: Involvement of PD-L1 on tumor cells in the escape from host immune system and tumor immunotherapy by PD-L1 blockade. Proc Natl Acad Sci USA 99: 12293-12297, 2002. 
11. Wilcox RA, Feldman AL, Wada DA, et al: B7-H1 (PD-L1, CD274) suppresses host immunity in T-cell lymphoproliferative disorders. Blood 114: 2149-2158, 2009.

12. Selenko-Gebauer N, Majdic O, Szekeres A, et al: B7-H1 (programmed death-1 ligand) on dendritic cells is involved in the induction and maintenance of T cell anergy. J Immunol 170: 3637-3644, 2003.

13. Fukaya T, Takagi H, Sato Y, et al: Crucial roles of B7-H1 and B7-DC expressed on mesenteric lymph node dendritic cells in the generation of antigen-specific $\mathrm{CD} 4^{+} \mathrm{Foxp} 3^{+}$regulatory T cells in the establishment of oral tolerance. Blood 116: 2266-2276, 2010.

14. Luo X, Tarbell KV, Yang H, et al: Dendritic cells with TGF-beta1 differentiate naive $\mathrm{CD} 4^{+} \mathrm{CD} 25^{-} \mathrm{T}$ cells into islet-protective Foxp3 $3^{+}$ regulatory T cells. Proc Natl Acad Sci USA 104: 2821-2826, 2007.

15. Yamazaki S, Bonito AJ, Spisek R, Dhodapkar M, Inaba K and Steinman RM: Dendritic cells are specialized accessory cells along with $\mathrm{TGF}^{-}$for the differentiation of $\mathrm{Foxp}^{+} \mathrm{CD}^{+}$regulatory T cells from peripheral Foxp3 precursors. Blood 110: 4293-4302, 2007.

16. Zhang C, Wu S, Xue X, et al: Antitumor immunotherapy by blockade of the PD-1/PD-L1 pathway with recombinant human PD-1-IgV. Cytotherapy 10: 711-719, 2008.

17. Dalum I, Jensen MR, Hindersson P, Elsner HI and Mouritsen S: Breaking of B cell tolerance toward a highly conserved self protein. J Immunol 157: 4796-4804, 1996.

18. Dalum I, Butler DM, Jensen MR et al: Therapeutic antibodies elicited by immunization against TNF-alpha. Nat Biotechnol 17: 666-669, 1999.
19. Xue X, Feng G, Li M, et al: Amelioration of experimental autoimmune encephalomyelitis by BLyS autovaccine. Vaccine 26: 2873-2881, 2008

20. Renard V, Sonderbye L, Ebbehoj K, et al: HER-2 DNA and protein vaccines containing potent $\mathrm{Th}$ cell epitopes induce distinct protective and therapeutic antitumor responses in HER-2 transgenic mice. J Immunol 171: 1588-1595, 2003.

21. Valmori D, Pessi A, Bianchi E and Corradin G: Use of human universally antigenic tetanus toxin $\mathrm{T}$ cell epitopes as carriers for human vaccination. J Immunol 149: 717-721, 1992.

22. Yu Z, Healy F, Valmori D, Escobar P, Corradin G and Mach JP: Peptide-antibody conjugates for tumour therapy: a MHC-class-IIrestricted tetanus toxin peptide coupled to an anti-Ig light chain antibody can induce cytotoxic lysis of a human B-cell lymphoma by specific CD4 T cells. Int J Cancer 56: 244-248, 1994.

23. Suh WK, Wang S, Duncan GS, et al: Generation and characterization of B7-H4/B7S1/B7x-deficient mice. Mol Cell Biol 26: 6403-6411, 2006.

24. Gallina G, Dolcetti L, Serafini P, et al: Tumors induce a subset of inflammatory monocytes with immunosuppressive activity on CD8 ${ }^{+}$T cells. J Clin Invest 116: 2777-2790, 2006.

25. Zang X and Allison JP: The B7 family and cancer therapy: costimulation and coinhibition. Clin Cancer Res 13: 5271-5279, 2007.

26. Blank C, Kuball J, Voelkl S, et al: Blockade of PD-L1 (B7-H1) augments human tumor-specific $\mathrm{T}$ cell responses in vitro. Int J Cancer 119: 317-327, 2006. 